\title{
Correlation of ventricular size and transcranial Doppler findings before and after ventricular peritoneal shunt in patients with hydrocephalus: prospective study of 35 patients
}

\author{
A Jindal, A K Mahapatra
}

\begin{abstract}
Objective-To compare the cerebral blood flow velocity and pulsatility index in patients with hydrocephalus and to correlate the blood flow velocity to shunt function and size of ventricles in CT. Methods-The study comprised 16 children and 19 adults, preoperative (transcranial Doppler, TCD) disclosed normal pulsatility index in six and raised pulsatility index in 29 patients. Preoperative CT showed moderate hydrocephalus in 32 and severe hydrocephalus in three patients. All patients had CT and TCD 36-48 hours after their shunt operation.

Results-A marked reduction in pulsatility index occurred postoperatively in 28 patients, all of them showed asignificant decrease in ventricular size on postoperative CT done simultaneously. Mild or no reduction of pulsatility index was recorded in seven patients and none of them showed decreased ventricular size on postoperative CT. In two patients subsequent shunt blockage was accompanied by appropriate changes in both pulsatility index and CT, which reversed after successful shunt revision.

Conclusion-Fall in pulsatility index correlated well with decrease in ventricle size. Thus TCD can be used as a simple, bedside test for the assessment of shunt function.

(F Neurol Neurosurg Psychiatry 1998;65:269-271)
\end{abstract}

All India Institute of Medical Sciences, New Delhi, Pin 110 029,

India

A Jindal

A K Mahapatra

Correspondence to: Professor A K Mahapatra, Department of

Neurosurgery, C N Centre, All India Institute of Medica Sciences, Ansari Nagar, New Delhi 110 029, India.

Received 23 May 1997 and in final revised form 14 November 1997

Accepted 2 Decenber 1997 anterior circulation were possible in $95 \%$ of the normal population and their normal values were reported. Transcranial Doppler does not give direct information about changes in cerebral blood flow, but a compromised flow velocity pattern in terms of raised pulsatility index (systolic velocity-diastolic velocity)/mean velocity) can be a sensitive index of impending ischaemic injury. ${ }^{6}$

Assessment of cerebral blood flow velocity alone in hydrocephalus using TCD can be unreliable and inaccurate due to an increased angle of insonation, where actual velocity may be a cosine function of velocity read by the transducer. In such a situation pulsatility index is a better index as it is a ratio of two velocities read by the same transducer and in calculating pulsatility index the error caused by the cosine function is cancelled. ${ }^{7}$ Therefore, a study was done to determine changes in pulsatility index and ventricle size after shunt to find out if the fall in pulsatility index correlated with decreased ventricle size.

\section{Materials and methods}

The study comprised 35 patients of whom 16 were children and 19 were adults (age range 8-75 years, 10 females and 25 males). These patients had hydrocephalus due to various aetiologies (posterior fossa tumour 27, suprasellar tumour two, posterior third ventricle tumour two, congenital hydrocephalus two). All patients had detailed clinical evaluation and had findings suggestive of raised intracranial pressure. Recordings from TCD were by the technique described by Aaslid et al. ${ }^{18}$ The temporal ultrasonic window, which lies above the zygomatic arch, was used and the patients were evaluated in a supine position. An EMETC 2B TCD machine was used with a $2 \mathrm{mHz}$ frequency probe and fast Fourier frequency analyser. In each patient the Doppler probe was positioned to overlie the pterion and was oriented within a few degrees parallel to the horizontal plane. The depth was adjusted to measure velocity in the middle cerebral artery and the internal carotid artery. These arteries were chosen because they are easier to insonate, the angle of insonation is 
Table 1 Statistical analysis

\begin{tabular}{|c|c|c|c|c|c|c|}
\hline & $n$ & Mean & $S D$ & Median & Minimum & Maximum \\
\hline \multicolumn{7}{|l|}{ Preoperative: } \\
\hline Group A & 28 & 1.056 & 0.324 & 0.94 & 0.70 & 2.25 \\
\hline Group B & 7 & 0.8957 & 0.197 & 0.92 & 0.56 & 1.15 \\
\hline \multicolumn{7}{|c|}{$\begin{array}{l}\text { Wilcoxon rank-sum test } z=0.969 \text {, probability }=0.1662(\mathrm{NS}) \\
\text { Preoperative-post-operative } \\
\text { change in PI: }\end{array}$} \\
\hline Group A & 28 & 0.378 & 0.232 & - & 0.14 & 1.27 \\
\hline Group B & 7 & 0.020 & 0.067 & - & 0.12 & 0.09 \\
\hline
\end{tabular}

Wilcoxon rank sum test $\mathrm{z}=4.041$, probability $=2.656 \mathrm{E}-0.5(\mathrm{p}<0.001)$.

small, and the frequency shift is assumed to reflect the true flow velocity. The normal value of the pulsatility index in the middle cerebral artery is reported as $0.66(0.06)$ (mean (SD)). A fall in pulsatility index $>0.12(>2 \mathrm{SD})$ was considered to be significant. Preoperative CT showed moderate hydrocephalus in 32 and severe hydrocephalus in three patients (Evans' index of preoperative CT). TheTCD study was repeated 36-48 hours after the shunt surgery and CT was obtained simultaneously. The correlation between the change in pulsatility index and change in ventricle size was determined. Patients were divided into two groups according to the fall in PT after surgery (group A, patients with a significant fall in pulsatility index; group B, patients with a non-significant fall in pulsatility index).

\section{Results}

Preoperative TCD showed a normal pulsatility index in six and a raised pulsatility index (above the normal value) in 29 patients.
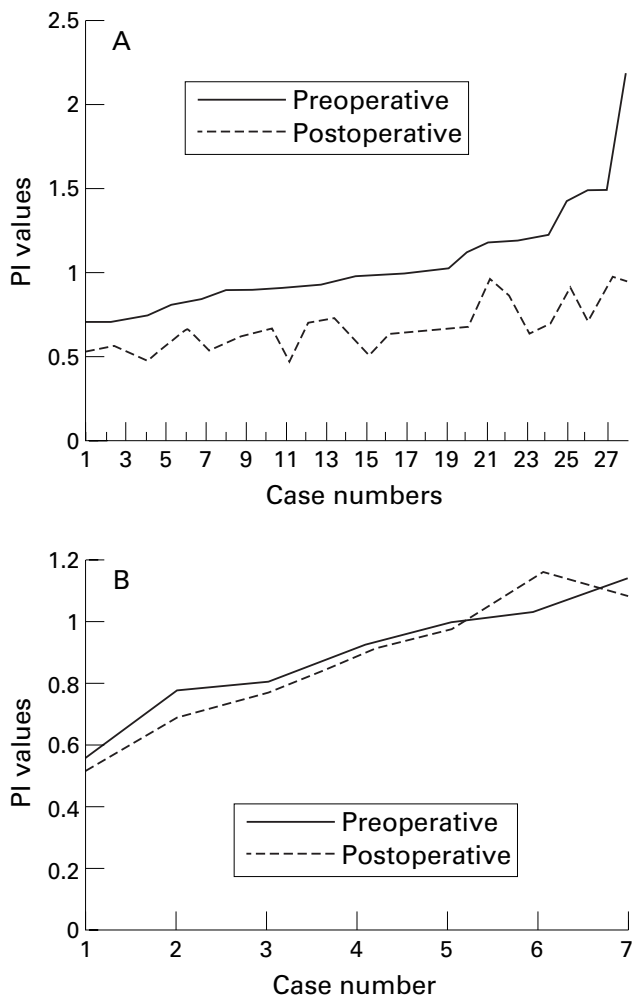

(A) Preoperative and postoperative pulsatility index values in group $A$ (in these patients ventricular size was reduced after shunt and pulsatility index values were also reduced significantly). (B) Preoperative and postoperative pulsatility index values in group $B$ (in these patients ventricular size was not reduced after shunt and pulsatility index values did not change significantly).
Hydrocephalus was moderate in 32 and severe in three patients. Postoperative pulsatility index values were reduced significantly in 28 patients (group A) and there was a nonsignificant reduction in seven (group B). Mean pulsatility index in group A was 1.056 (SD 0.324 ) whereas that in group $B$ was 0.896 (SD 0.197 ) (preoperative). This difference was not significant. A fall in pulsatility index in group A patients was 0.378 (SD 0.232) and reduction in pulsatility index values postoperatively in group B was 0.02 (SD 0.067). All patients in group A showed ventricle size to be decreased to normal on postoperative CT and none of the group B patients had changes in the degree of hydrocephalus. Changes in pulsatility index in the two groups were compared using a Wilcoxon rank sum test and were significant (table; $p<0.001$ ). Two patients from group A during follow up for their primary pathology developed shunt malfunction and were readmitted. Brain CT showed hydrocephalus and pulsatility index values on TCD were high on admission. After shunt revision both ventricle size and pulsatility index values returned to normal. Group A values for pulsatility index preoperatively and the preoperative-postoperative change in pulsatility index were not normally distributed (coefficients of skewness and kurtosis were calculated and deviation from normality was confirmed using a $\mathrm{z}$ test), justifying the use of a non-parametric test for statistical analysis (the figure shows the pulsatility index values in the two groups).

\section{Discussion}

During the past decade continuous and pulsed wave Doppler have been used extensively for accurate evaluation of extracranial arterial obstructive lesions; however, the use of Doppler in assessing intracranial arterial flow is not commonly practised. Hasseler et al described changes in cerebral blood flow $(\mathrm{CBF})$ velocity with increasing intracranial pressure (ICP). Diastolic velocity is affected first and with progressive rise in ICP diastolic velocity decreases and pulsatility index increases. Hill et $a l^{1}$ suggested that among ventriculomegaly and raised ICP, ventriculomegaly is a more important factor in the genesis of a raised pulsatility index. Wozniak et $a l^{3}$ reported experimental evidence showing an adverse effect of ventriculomegaly on $\mathrm{CBF}$, apparently by displacement, deformation, stretching, and decrease in the calibre of cerebral arteries and also demonstrated dilatation of cerebral blood vessels after ventricular drainage. Weir et $a l^{10}$ showed a pro- 
gressive decrease in CBF with increase in ventricle size. Quinn et $a l^{11}$ recorded the pulsatility index in children with blocked shunts and showed that a raised pulsatility index in such patients returned to values comparable with the control group after shunt revision. However, all the patients operated on did not have postoperative CT for correlation. Moreover, all these patients did not have ICP recordings preoperatively and postoperatively to allow a conclusion as to whether a fall in ICP or decreased ventricle size correlated with fall in pulsatility index. Nadvi et $a l^{12}$ used TCD as a non-invasive method for evaluating shunt function and found a significant reduction in pulsatility index after shunt. Their study included only children and no postoperative CT was carried out to document shunt function. In our study postoperative CT was carried out in all patients and pulsatility index was correlated with a fall in ventricle size. Our study also included both children and adults, suggesting that a fall in pulsatility index correlates with change in ventricle size in all age groups whereas earlier studies have been done only in children. Studies on TCD in untreated hydrocephalus have shown raised a pulsatility index but variable results have been obtained for systolic, mean, and diastolic flow velocity. This is probably due to vessel distortion, which may increase the angle of insonation. ${ }^{7}$ In our study 29 of 35 patients had a raised pulsatility index before shunt surgery. The lack of specificity of symptoms of shunt blockage has resulted in greater reliance on the examination and investigation. Palpation or pumping of the chamber is unreliable. Aspiration of the reservoir may be helpful if the distal catheter is blocked but carries the risk of introducing infection and damaging the chamber. ${ }^{11}$ Brain CT shows enlarged ventricles but a previous scan is required for comparison. Two of our patients who were readmitted with clinical evidence of a blocked shunt showed a significant rise in pulsatility index values. Further CT involves a moderate dose of ionising radiation and moving the patient to the CT room. In view of this TCD provides a non-invasive way of determining changes in ventricle size. It is inexpensive, portable, and can be repeated as often as required without any radiation exposure. It should be noted that results of TCD are limited by the knowledge, skill, and experience of the person performing it. Our study has shown a good correlation of shunt function and pulsatility index values.

\section{Conclusion}

Changes in pulsatility index values after shunt have a significant correlation with change in ventricle size. Therefore, pulsatility index as measured by TCD is a good indicator of shunt function. TCD is a simple, non-invasive, bedside test which can be used to evaluate shunt blockage.

1 Hill A, Volpe JJ. Decrease in pulsatile flow in ACA in infantile hydrocephalus. Paediatrics 1982;69:4-7.

2 Lui H, Hillman J, Sprigg A, et al. CBF velocity patterns in posthemorrhagic ventricular dilation. Child Nervs Syst 1990;6:250-3

3 Wozniak M, Mclane DC, Raimondi AJ. Micro and macro vascular changes as a direct cause of congenital murine hydrocephalus. $\mathcal{F}$ Neurosurg. 1975;48:530-5.

4 Kety SS, Shenkir HA, Schmidt CF. The effect of increased $\mathrm{CP}$ in cerebral circulatory function in man. 7 Clin Invest 1948;27:493-9.

5 Aaslid R, Markwalder TM, Normes H. Non-invasive TCD ultrasound recording of flow velocity in basal cerebral trasound recording of flow velocity

6 Gosling. F Paraycheu T, Kirkham F Transcranial measurement of blood flow velocity in the basal cerebral arteries using pulsed Doppler ultrasound-a method of arteries using pulsed Doppler ultrasound-a method of 14.

14

7 Finn P, Quinn MOO, Hall-Craggs MA. Vessel distortion may invalidate TCD velocity measurements, MRI correlation. F Neurosurg. 1990;73:572-5.

8 Lindegaard KF, Bakke SJ, Grolimund P, et al. Assessment of intracranial haemodynamics in carotid artery disease by TCD ultrasound. $\mathcal{F}$ Neurosurg 1985;63:980-8.

9 Hassler W, Steinmetz H, Gawlowski J. TCD ultrasonography in raised ICP and in intracranial circulatory arrest. $\mathcal{f}$ Neurosurg 1988;68:745-1.

10 Weir B, Menon D, Oreeton T. Regional CBF in patient with aneurysm-estimation by xenon 133 inhalation. Can $\mathcal{F ~ N e u -}$ rolog Sci 1978;2:301-5.

11 Quinn MV, Pople JK. MCA pulsatility in children with blocked CSF shunt. I Neurol Neurosurg Psychiatry 1992;55: 525-7.

12 Nadvi SS, Du Trevou MD, Vandellen JR, et al. The use of TCD ultrasonography as a method of assessing ICP in hydrocephalic children. Br F Neurosurg 1994;8:573-7. 